\title{
Convergence of Dirichlet eigenvalues for elliptic systems on perturbed domains
}

\author{
Justin L. Taylor
}

Abstract. We consider the eigenvalues of an elliptic operator

$$
(L u)^{\beta}=-\frac{\partial}{\partial x_{j}}\left(a_{i j}^{\alpha \beta} \frac{\partial u^{\alpha}}{\partial x_{i}}\right), \quad \beta=1, \ldots, m,
$$

where $u=\left(u^{1} \ldots, u^{m}\right)^{t}$ is a vector valued function and $a^{\alpha \beta}(x)$ are $(n \times n)$ matrices whose elements $a_{i j}^{\alpha \beta}(x)$ are at least uniformly bounded measurable real-valued functions such that

$$
a_{i j}^{\alpha \beta}(x)=a_{j i}^{\beta \alpha}(x)
$$

for any combination of $\alpha, \beta, i$, and $j$. We assume we have two non-empty, open, disjoint, and bounded sets, $\Omega$ and $\widetilde{\Omega}$, in $\mathbb{R}^{n}$, and add a set $T_{\varepsilon}$ of small measure to form the domain $\Omega_{\varepsilon}$. Then we show that as $\varepsilon \rightarrow 0^{+}$, the Dirichlet eigenvalues corresponding to the family of domains $\left\{\Omega_{\varepsilon}\right\}_{\varepsilon>0}$ converge to the Dirichlet eigenvalues corresponding to $\Omega_{0}=\Omega \cup \widetilde{\Omega}$. Moreover, our rate of convergence is independent of the eigenvalues. In this paper, we consider the Lamé system, systems which satisfy a strong ellipticity condition, and systems which satisfy a Legendre-Hadamard ellipticity condition.

Mathematics Subject Classification (2010). 35, 43.

Keywords. Eigenvalues, elliptic systems, perturbed domains.

\section{Introduction}

There is a great deal of work studying eigenvalues for elliptic equations, but there seems to be less work on eigenvalues for elliptic systems. Much of the work on equations requires estimates for solutions that do not hold for systems. In this paper, we consider the behavior of eigenvalues for elliptic systems in singularly perturbed domains. We give a simple characterization of the families of domains that we can study and this class includes families such as dumbbell domains formed by connecting two domains by a thin tube. We show that as the measure of the perturbation shrinks away, the convergence of the eigenvalues is obtained. We also provide a rate of convergence, which is independent of any eigenvalue. We make no assumption on 
the smoothness of the coefficients and only mild assumptions on the boundary of the domain.

Studying solutions of elliptic boundary value problems with Dirichlet or Neumann boundary conditions on domains which can be approximated by solutions on simpler domains has been an interest for many years, and is still ongoing. The motivation to study such problems is that it is easier to study the spectra on sets with a reduced dimensionality. One may approximate the spectra on these "fattened" sets with the spectra on the "thinner" sets. Some applications include studying quantum wires, free-electron theory of conjugated molecules, and photonic crystals. For a complete description, see the work of Kuchment [26]. Recent work by Exner and Post [15] study the Neumann Laplacian on manifolds with thin tubes which is related to the theory of quantum graphs. The Fireman's Pole problem consists of approximating the resolvents of a bounded set in $\mathbb{R}^{3}$ by the resolvents of this set with a cylinder removed. For a complete description, see Rauch and Taylor [29]. A classic paper by Babuska and Výborný [5] shows continuity of Dirichlet eigenvalues for elliptic equations under a regular variation of the domain, but gives no rates of convergence. Dancer [11], [12] considers how perturbing the domain affects the number of positive solutions for nonlinear equations with Dirichlet boundary conditions and includes the case where solutions are eigenfunctions for the Laplacian. Davies [14] and Pang [28] study the approximation of Dirichlet eigenvalues and corresponding eigenfunctions in a domain $\Omega$ by eigenvalues and eigenfunctions in sets of the form $R(\varepsilon)=\{x \in$ $\Omega$ : $\operatorname{dist}(x, \partial \Omega) \geq \varepsilon\}$. They each give rates of convergence and their estimates include the case when the domain is irregularly shaped. The work of Brown, Hislop, and Martinez [6] provides upper and lower bounds on the splitting between the first two Dirichlet eigenvalues in a symmetric dumbbell region with a straight tube. Chavel and Feldman [9] examine eigenvalues on a compact manifold with a small handle and Dirichlet conditions on the ends of the handle. The work of Anné and Colbois [1] examines the behavior of eigenvalues of the Laplacian on $p$-forms under a singular perturbation obtained by adding a thin handle to a compact manifold, but requires more regularity on the eigenfunctions than holds in our setting.

More recent work for Dirichlet conditions includes work by Daners [13], which shows convergence of solutions for elliptic equations on sequences of domains. These domains $\Omega_{n}$ converge to a limit domain $\Omega$ in the sense of sequences $u_{n} \in H_{0}^{1}\left(\Omega_{n}\right)$ converging to a function $u \in H_{0}^{1}(\Omega)$. Also, Burenkov and Lamberti [8] prove sharp spectral stability estimates for higher-order elliptic operators on domains in certain Hölder classes in terms of the Lebesgue measure of the symmetric difference of the different domains. Kozlov [25] obtains asymptotics of Dirichlet eigenvalues for domains in $\mathbb{R}^{n}$ for $n \geq 2$ using Hadamard's formula. Grieser and Jerison [20] also give asymptotics for Dirichlet eigenvalues and eigenfunctions, but only on plane domains.

We note here that the results for Neumann eigenvalues may be different than those for Dirichlet eigenvalues. In fact, a classic example of Courant and Hilbert [10] shows that the Neumann eigenvalues may not vary continuously as the domain varies. Their 
example is constructed by taking the unit square in $\mathbb{R}^{2}$ and attaching a thin handle with a proportional square attached to the other end. They show that if $\left\{\lambda_{n}^{\varepsilon}\right\}$ and $\left\{\lambda_{n}^{0}\right\}$ are the Neumann eigenvalues of $-\Delta$ in increasing order including multiplicities with respect to the perturbed square and the unit square, then $\lambda_{2}^{\varepsilon} \rightarrow 0$ as $\varepsilon \rightarrow 0$, but $\lambda_{2}^{0}>0$. This example shows that one needs additional regularity in order to achieve convergence. Furthermore, Arrieta, Hale, and Han [4] show that for this type of domain, $\lambda_{m}^{\varepsilon} \rightarrow \lambda_{m-1}^{0}$, as $\varepsilon \rightarrow 0$ for $m \geq 3$. Another work of Arrieta [3] gives rates of convergence for eigenvalues of the Neumann Laplacian on a dumbbell domain in $\mathbb{R}^{2}$ when the tube is more general. Jimbo and Morita [24] study the first $N$ eigenvalues of the Neumann Laplacian in $N$ disjoint domains connected by thin tubes. They show that the first $N$ eigenvalues approach zero and the $(N+1)$ st eigenvalue is uniformly bounded away from zero. If $D_{1}$ and $D_{2}$ are two disjoint domains, then for $\left\{\sigma_{k}\right\}=\left\{\mu_{l}\right\} \cup\left\{\lambda_{j}\right\}$, where $\left\{\mu_{l}\right\}$ are the Neumann eigenvalues of $-\Delta$ in $D=D_{1} \cup D_{2}$ and $\left\{\lambda_{j}\right\}$ are the Dirichlet eigenvalues of $\frac{-d^{2}}{d x^{2}}$ in $(-1,1)$, Jimbo [22] gives a rate of convergence on the difference $\sigma_{k}-\sigma_{k}^{\varepsilon}$. This work was generalized to more classes of domains in a more recent work by Jimbo and Kosugi [23]. Also, Brown, Hislop, and Martinez [7] show that if $\sigma_{k} \in\left\{\mu_{l}\right\} \backslash\left\{\lambda_{j}\right\}$ then

$$
\left|\sigma_{k}-\sigma_{k}^{\varepsilon}\right| \leq C\left[\log \left(\frac{1}{\varepsilon}\right)\right]^{\frac{-1}{2}}, \quad n=2,
$$

and

$$
\left|\sigma_{k}-\sigma_{k}^{\varepsilon}\right| \leq C \varepsilon^{\frac{n-2}{2}}, \quad n \geq 3 .
$$

Here, we aim to provide an outline of the proof. In section 2, we give several definitions and describe the family of domains for which we can prove the convergence of eigenvalues. We also describe the well-known construction of eigenvalues and state our main result. In section 3, we give Theorem 3.1 from Giaquinta and Modica [17] and [18] which uses a technique introduced by Gehring [16]. We also prove a Caccioppoli type estimate for eigenfunctions in Theorem 3.4 and use this along with Theorem 3.1 to obtain a reverse Hölder inequality given in Theorem 3.5. This gives $L^{p}$-integrability for the gradient of the eigenfunctions for $p>2$. In section 4 , we are able to bound these $L^{p}$ norms by a constant in Proposition 4.2. The proof uses the reverse Hölder inequality as the key ingredient. This estimate is then used to prove Lemma 4.2 and Proposition 4.4, which are used to satisfy the first part of a well-known theorem from Anné [2] given in Lemma 4.1. The second part of Lemma 4.1 follows from the first part along with the above estimates, thus giving Corollary 4.1. The main result follows from this corollary. As a by-product of our research, we give a simple proof of Shi and Wright's [30] $L^{p}$-estimates for the gradient of the Lamé system as well as other elliptic systems. Many of the results first appeared in the author's Ph.D. dissertation [31].

Acknowledgments. The author thanks Russell Brown for his valuable discussions and suggestions. The author also thanks the referee for his or her helpful comments. 


\section{Preliminaries and main result}

We give conditions on a family of domains $\Omega_{\varepsilon}$ that allow us to prove the convergence of eigenvalues. We let $\Omega$ and $\widetilde{\Omega}$ in $\mathbb{R}^{n}$ be two non-empty, open, disjoint, and bounded sets. We let $\varepsilon_{1}>0$ (which will be chosen small later), and then let $\left\{T_{\varepsilon}\right\}_{0<\varepsilon \leq \varepsilon_{1}}$ be a family of open sets such that

$$
T_{\tilde{\varepsilon}} \subset T_{\varepsilon} \text { if } \tilde{\varepsilon} \leq \varepsilon
$$

If $\left|T_{\varepsilon}\right|$ denotes the Lebesgue measure of $T_{\varepsilon}$, then

$$
\left|T_{\varepsilon}\right| \leq C \varepsilon^{d},
$$

where $C$ and $0<d \leq n$ are independent of $\varepsilon$. Fix two points $p_{1}$ and $p_{2}$ on $\partial \Omega$ and $\partial \widetilde{\Omega}$, respectively. For each $\varepsilon$, let $B_{\varepsilon}$ and $\widetilde{B}_{\varepsilon}$ be two balls of radius $\varepsilon$ in $\mathbb{R}^{n}$ centered at $p_{1}$ and $p_{2}$, respectively. The connections from $T_{\varepsilon}$ to $\Omega$ and $\widetilde{\Omega}$ will be contained in $B_{\varepsilon}$ and $\widetilde{B}_{\varepsilon}$, so that $T_{\varepsilon} \cap \Omega=\emptyset$ and $\bar{T}_{\varepsilon} \cap \bar{\Omega} \subset B_{\frac{\varepsilon}{2}}$ where $B_{\frac{\varepsilon}{2}}$ is the concentric ball to $B_{\varepsilon}$ of radius $\frac{\varepsilon}{2}$. Also, suppose a similar condition for $\widetilde{\Omega}$ and $\widetilde{B}_{\varepsilon}$. Then for any $\varepsilon$, define $\Omega_{\varepsilon}$ to be the set $\Omega \cup \widetilde{\Omega} \cup T_{\varepsilon}$, which we assume to be open and connected, and $\Omega_{0}=\Omega \cup \widetilde{\Omega}$. So, if our family is the family of dumbbell domains, you may think of $T_{\varepsilon}$ as a "tube" connecting each of the two domains. We now have the family of domains $\left\{\Omega_{\varepsilon}\right\}_{0 \leq \varepsilon \leq \varepsilon_{1}}$.

Next, we give a condition on the boundary of $\Omega_{\varepsilon}$. If $B_{r}$ is any ball of radius $r$ satisfying $B_{r} \cap \Omega_{\varepsilon}^{c} \neq \emptyset$, then

$$
\left|B_{2 r} \cap \Omega_{\varepsilon}^{c}\right| \geq C_{0} r^{n},
$$

where $C_{0}$ is a constant independent of $r$ and $\varepsilon$. This eliminates domains with "cracks" and "in-cusps," and will be used to help show the Caccioppoli inequality in Theorem 3.4 for the case when we are close to the boundary.

Throughout this paper we use the convention of summing over repeated indices, where $i$ and $j$ will run from 1 to $n$ and $\alpha, \beta$, and $\gamma$ will run from 1 to $m$. We let $a_{i j}^{\alpha \beta}(x)$ be bounded, measurable, real-valued functions on $\mathbb{R}^{n}$ which satisfy the symmetry condition

$$
a_{i j}^{\alpha \beta}(x)=a_{j i}^{\beta \alpha}(x), \quad i, j=1,2, \ldots, n, \alpha, \beta=1,2, \ldots, m .
$$

We let $L^{2}\left(\Omega_{\varepsilon}\right)$ denote the space of square integrable functions taking values in $\mathbb{R}^{m}$ and $H_{0}^{1}\left(\Omega_{\varepsilon}\right)$ denotes the Sobolev space of vector-valued functions having one derivative in $L^{2}\left(\Omega_{\varepsilon}\right)$ and which vanish on the boundary. We use $u_{j}^{\alpha}$ to denote the partial derivative $\frac{\partial u^{\alpha}}{\partial x_{j}}$. 
Let $\eta_{\varepsilon} \in C_{c}^{\infty}\left(\mathbb{R}^{n}\right)$ be a cutoff function so that we have $\eta_{\varepsilon}=0$ in $T_{\varepsilon}, \eta_{\varepsilon}=1$ in $\Omega_{0} \backslash\left(B_{\varepsilon} \cup \widetilde{B}_{\varepsilon}\right),\left|\nabla \eta_{\varepsilon}\right| \leq \frac{C_{n}}{\varepsilon}$, and $0 \leq \eta_{\varepsilon} \leq 1$, where $C_{n}$ only depends on $n$. We emphasize that $B_{\varepsilon}, \widetilde{B}_{\varepsilon}$, and $\eta_{\varepsilon}$ depend on the parameter $\varepsilon$. With these assumptions and definitions, we have that for any $u \in H_{0}^{1}\left(\Omega_{\varepsilon}\right), \eta_{\varepsilon} u$ will be in $H_{0}^{1}\left(\Omega_{0}\right)$.

We now introduce the notion of an eigenvalue and corresponding eigenvector. We say that the number $\sigma$ is a Dirichlet eigenvalue of $L$ with Dirichlet eigenfunction $u \in H_{0}^{1}(\Omega)$, if $u \neq 0$ and

$$
\int_{\Omega} a_{i j}^{\alpha \beta}(x) u_{i}^{\alpha}(x) \varphi_{j}^{\beta}(x) d x=\sigma \int_{\Omega} u^{\gamma}(x) \varphi^{\gamma}(x) d x, \quad \varphi \in H_{0}^{1}(\Omega) .
$$

We say that $L$ satisfies the Legendre-Hadamard condition if there exists $\theta>0$ so that

$$
a_{i j}^{\alpha \beta}(x) \xi_{\alpha} \xi_{\beta} \psi_{i} \psi_{j} \geq \theta|\xi|^{2}|\psi|^{2}, \quad \xi \in \mathbb{R}^{m}, \psi \in \mathbb{R}^{n} \text {, a.e. } x \in \Omega_{\varepsilon} \text {. }
$$

If we define the norm on matrices $A=A_{i j} \in \mathbb{R}^{m \times n}$ as

$$
|A|^{2}=\sum_{i=1}^{m} \sum_{j=1}^{n}\left|A_{i j}\right|^{2}
$$

and $L$ satisfies the Legendre-Hadamard condition with continuous coefficients in $\bar{\Omega}$, then it is well-known that for any $u \in H_{0}^{1}(\Omega)$, we have Gårding's inequality [32], p. 347 ,

$$
C_{1} \int_{\Omega}|\nabla u|^{2} d x \leq \int_{\Omega} a_{i j}^{\alpha \beta}(x) u_{i}^{\alpha}(x) u_{j}^{\beta}(x) d x+C_{2} \int_{\Omega}|u|^{2} d x .
$$

$L$ is said to satisfy a strong Legendre condition or a strong ellipticity condition if there exists $\theta>0$ so that

$$
a_{i j}^{\alpha \beta}(x) \xi_{i}^{\alpha} \xi_{j}^{\beta} \geq \theta|\xi|^{2}, \quad \xi \in \mathbb{R}^{m \times n} \text {, a.e. } x \in \Omega_{\varepsilon} .
$$

We introduce the Lamé system as $L u=-\operatorname{div} \zeta(u)$, where $\zeta(u)$ denotes the stress tensor defined by

$$
\zeta_{j}^{\beta}(u) \stackrel{\text { def }}{=} a_{i j}^{\alpha \beta} u_{i}^{\alpha}
$$

which is defined in terms of the Lamé moduli $v(x)$ and $\mu(x)$ by

$$
a_{i j}^{\alpha \beta}(x) \stackrel{\text { def }}{=} v(x) \delta_{i \alpha} \delta_{j \beta}+\mu(x) \delta_{i j} \delta_{\alpha \beta}+\mu(x) \delta_{i \beta} \delta_{j \alpha},
$$

where $v(x)$ and $\mu(x)$ are both assumed to be bounded and measurable. Also, define the strain tensor $\kappa(u)$ as

$$
\kappa_{i j}(u) \stackrel{\text { def }}{=} \frac{1}{2}\left(u_{j}^{i}+u_{i}^{j}\right) .
$$


Note that for the Lamé system, $m=n$ and the Lamé parameters $v(x)$ and $\mu(x)$ given in (2.8) satisfy the conditions

$$
v(x) \geq 0 \text { and } \mu(x) \geq \delta>0 .
$$

With these assumptions, the Lamé system satisfies the ellipticity condition

$$
a_{i j}^{\alpha \beta} u_{i}^{\alpha} u_{j}^{\beta} \geq \tau|\kappa(u)|^{2}, \quad u \in H_{0}^{1}\left(\Omega_{\varepsilon}\right)
$$

where $\tau=2 \delta$. With Korn's 1st Inequality, it is easy to see that for the Lamé system, we have

$$
\frac{\tau}{2} \int_{\Omega_{\varepsilon}}|\nabla u|^{2} d y \leq \int_{\Omega_{\varepsilon}} a_{i j}^{\alpha \beta} u_{i}^{\alpha} u_{j}^{\beta} d y, \quad u \in H_{0}^{1}\left(\Omega_{\varepsilon}\right) .
$$

Thus, if u satisfies either the ellipticity condition (2.6), (2.11), or (2.4) with continuous coefficients in $\bar{\Omega}$, then we have Gårding's inequality (2.5).

The well-known construction of eigenvalues and eigenfunctions for scalar functions (which is the same for vector-valued functions) is taken from Gilbarg and Trudinger [19], p. 212. If we define the bilinear form on $H_{0}^{1}\left(\Omega_{\varepsilon}\right) \times H_{0}^{1}\left(\Omega_{\varepsilon}\right)$ as

$$
B_{\varepsilon}(u, v) \stackrel{\text { def }}{=} \int_{\Omega_{\varepsilon}} a_{i j}^{\alpha \beta} u_{i}^{\alpha} v_{j}^{\beta} d x
$$

and define the Rayleigh quotient $R_{\varepsilon}$ as

$$
R_{\varepsilon}(u) \stackrel{\text { def }}{=} \frac{B_{\varepsilon}(u, u)}{\|u\|_{L^{2}\left(\Omega_{\varepsilon}\right)}^{2}}
$$

for $u \neq 0$, then we can construct an increasing sequence of eigenvalues, listed according to multiplicity, $\left\{\sigma_{k}\right\}_{k=1}^{\infty}$ such that for each corresponding eigenfunction $u_{k} \in H_{0}^{1}\left(\Omega_{\varepsilon}\right)$, we have

$$
\min _{w \in\left\{u_{1}, \ldots, u_{k-1}\right\}^{\perp}} R_{\varepsilon}(w)=R_{\varepsilon}\left(u_{k}\right)=\sigma_{k}
$$

and

$$
\left\|u_{k}\right\|_{L^{2}\left(\Omega_{\varepsilon}\right)}=1
$$

for any $k$. Furthermore, each eigenspace is finite-dimensional and the constructed set of eigenfunctions forms an orthonormal basis in $L^{2}\left(\Omega_{\varepsilon}\right)$.

We now state the main result.

Theorem 2.1. Let

$$
(L u)^{\beta}=-\frac{\partial}{\partial x_{j}}\left(a_{i j}^{\alpha \beta} \frac{\partial u^{\alpha}}{\partial x_{i}}\right) \quad \beta=1, \ldots, m
$$

satisfy one of the following conditions: 
(1) L has uniformly bounded coefficients and satisfies either the ellipticity condition (2.6) or the ellipticity condition (2.11);

(2) L has continuous coefficients and satisfies the ellipticity condition (2.4).

Also assume $\left\{\sigma_{k}^{0}\right\}_{k=1}^{\infty}$ and $\left\{\sigma_{k}^{\varepsilon}\right\}_{k=1}^{\infty}$ are the Dirichlet eigenvalues of $L$ with respect to $\Omega_{0}$ and $\Omega_{\varepsilon}$ in increasing order numbered according to multiplicity. Then for each $J \in \mathbb{N}$, we have the following estimate:

$$
\left|\sigma_{J}^{\varepsilon}-\sigma_{J}^{0}\right| \leq C \varepsilon^{a}
$$

for $0<\varepsilon \leq \varepsilon_{0}(J)$, where $\varepsilon_{0}(J)$ depends on the multiplicity of $\sigma_{J}^{0}$. Moreover, the rate $a>0$ is independent of any eigenvalue and $C$ only depends on the eigenvalue $\sigma_{J}^{0}$ and the distance from $\sigma_{J}^{0}$ to nearby eigenvalues.

\section{A reverse Hölder inequality}

If

$$
f_{E}|f(y)| d y
$$

is defined to be the average of $f$ on $E$, then recall that the maximal function is defined for $f \in L_{\text {loc }}^{1}\left(\mathbb{R}^{n}\right)$ to be

$$
M(f)(x) \stackrel{\text { def }}{=} \sup _{r>0} f_{B_{r}(x)}|f(y)| d y
$$

where $B_{r}(x)$ is a ball of radius $r$ centered at $x$. Also, define $M_{R}(f)(x)$ to be

$$
M_{R}(f)(x) \stackrel{\text { def }}{=} \sup _{R>r>0} f_{B_{r}(x)}|f(y)| d y .
$$

We will need the following theorem from Giaquinta [17], p. 122, which uses the technique introduced by Gehring [16], and refined by Giaquinta and Modica [18].

Theorem 3.1. Let $r>q>1$, and $Q_{R}$ be a cube in $\mathbb{R}^{n}$ with sidelength $R$ centered at 0 . Define

$$
d(x)=\operatorname{dist}\left(x, \partial Q_{R}\right) .
$$

If $f$ and $g$ are measurable functions such that $f \in L^{r}\left(Q_{R}\right), g \in L^{q}\left(Q_{R}\right), f=$ $g=0$ outside $Q_{R}$, and with the added condition that

$$
M_{\frac{d(x)}{2}}\left(|g|^{q}\right)(x) \leq b M^{q}(g)(x)+M\left(|f|^{q}\right)+a M\left(|g|^{q}\right)(x)
$$

for almost every $x$ in $Q_{R}$ where $b \geq 0$ and $0 \leq a<1$, then $g \in L^{p}\left(Q_{\frac{R}{2}}\right)$, for $p \in[q, q+\varepsilon)$ and

$$
\left(f_{Q_{R / 2}}|g|^{p}(y) d y\right)^{\frac{1}{p}} \leq C\left[\left(f_{Q_{R}}|g|^{q}(y) d y\right)^{\frac{1}{q}}+\left(f_{Q_{R}}|f|^{p}(y) d y\right)^{\frac{1}{p}}\right]
$$

where $\varepsilon$ and $C$ depend on $b, q, n, a$, and $r$. 
The conclusion of this theorem is known as a reverse Hölder inequality. To show that the gradient of eigenfunctions satisfy this inequality, we will need to prove a Caccioppoli inequality. However, to show this Caccioppoli inequality, we first need the following two well-known inequalities taken from Hebey [21], p. 44, and Oleinik [27], p. 27.

Theorem 3.2 (Sobolev-Poincaré Inequality). Let $1 \leq p<n$ and $\frac{1}{q}=\frac{1}{p}-\frac{1}{n}$. Also, let $B_{r}$ be any ball of radius $r$ with $u \in W^{1, p}\left(B_{r}\right)$. Then, for $S$ contained in $B_{r}$ with $|S| \geq c_{0} r^{n}$,

$$
\int_{B_{r}}\left|u(x)-u_{S}\right|^{q} d x \leq C\left(\int_{B_{r}}|\nabla u|^{p}(x) d x\right)^{\frac{q}{p}}
$$

where $u_{S}=\frac{1}{|S|} \int_{S} u d y$, for some constant $C\left(n, p, c_{0}\right)$, independent of $u$.

Theorem 3.3 (Korn's Inequality on a Ball). If $u \in H^{1}\left(B_{r}\right)$ then

$$
\|\nabla u\|_{L^{2}\left(B_{r}\right)}^{2} \leq C\left(\|\kappa(u)\|_{L^{2}\left(B_{r}\right)}^{2}+\frac{1}{r^{2}}\|u\|_{L^{2}\left(B_{r}\right)}^{2}\right)
$$

where $C$ only depends on $n$.

We now state and prove a Caccioppoli inequality for eigenfunctions.

Theorem 3.4. Let $u$ be an eigenfunction with eigenvalue $\sigma$ associated to the operator $L$ satisfying either (2.6) or (2.11) with uniformly bounded coefficients or associated to (2.4) with continuous coefficients. Extending $u$ to be 0 outside $\Omega_{\varepsilon}$, there exists $r_{0}>0$ so that if $r_{0} \geq r>0, x \in \mathbb{R}^{n}$, we have

$$
\begin{aligned}
f_{B_{r}}|\nabla u|^{2} d y \leq & C_{1}\left(f_{B_{2 r}}|\nabla u|^{\frac{2 n}{n+2}} d y\right)^{\frac{n+2}{n}} \\
& +C_{2}|\sigma| f_{B_{2 r}}|u|^{2} d y+C_{3} f_{B_{2 r}}|\nabla u|^{2} d y
\end{aligned}
$$

where $B_{r}$ is a ball with radius $r$ centered at $x, C_{3}<1$, and $C_{l}>0$ only depends on $M=\max _{i, j, \alpha, \beta}\left\|a_{i j}^{\alpha \beta}\right\|_{L^{\infty}\left(\Omega_{\varepsilon}\right)}, n, m, \theta, \tau$, and $C_{0}$. Furthermore, if $L$ satisfies either (2.6) or (2.11) with uniformly bounded coefficients, then the inequality holds for any $r>0$.

Proof. First, choose a ball $B_{r}$ and define a cutoff function $v \in C_{c}^{\infty}\left(\mathbb{R}^{n}\right)$ to be so that $v=1$ in $B_{r}, v=0$ outside $B_{2 r},|\nabla v| \leq \frac{C_{n}}{r}$, and $0 \leq v \leq 1$, where $C_{n}$ only depends on $n$. Below, we will find an appropriate constant vector $\rho \in \mathbb{R}^{m}$, so that $v^{2}(u-\rho) \in H_{0}^{1}\left(\Omega_{\varepsilon}\right)$. By the weak formulation (2.3), we have

$$
\int_{\Omega_{\varepsilon}} a_{i j}^{\alpha \beta} u_{i}^{\alpha}\left[v^{2}(u-\rho)\right]_{j}^{\beta} d y=\sigma \int_{\Omega_{\varepsilon}} u^{\gamma}\left[v^{2}(u-\rho)\right]^{\gamma} d y .
$$


Then, performing the differentiations, we get

$$
\int_{\Omega_{\varepsilon}} a_{i j}^{\alpha \beta} u_{i}^{\alpha}\left[2 v v_{j}(u-\rho)^{\beta}+v^{2} u_{j}^{\beta}\right] d y=\sigma \int_{\Omega_{\varepsilon}} u^{\gamma} v^{2}(u-\rho)^{\gamma} d y .
$$

From this point, the argument depends on the ellipticity condition. We have three cases.

Case 1: $L$ satisfies the strong ellipticity condition (2.6). Using (2.6) and properties of $v$, we obtain the inequality

$$
\int_{B_{2 r}} v^{2} a_{i j}^{\alpha \beta} u_{i}^{\alpha} u_{j}^{\beta} d y \leq \int_{B_{2 r}} 2 M \frac{C_{n}}{r} \nu\left|\nabla u\left\|u-\rho\left|d y+\int_{B_{2 r}}\right| \sigma|| u\right\| u-\rho\right| d y
$$

which, for any constant $\omega>0$, then leads to

$$
\begin{gathered}
\int_{B_{2 r}} v^{2} a_{i j}^{\alpha \beta} u_{i}^{\alpha} u_{j}^{\beta} d y \leq \int_{B_{2 r}} \frac{\omega v^{2}|\nabla u|^{2}}{2} d y+\frac{C}{\omega r^{2}} \int_{B_{2 r}}|u-\rho|^{2} d y \\
+C|\sigma| \int_{B_{2 r}}|u|^{2} d y
\end{gathered}
$$

where $C$ depends on $M$ and $C_{n}$. Then choosing $\omega=\theta$ in (3.6) gives

$$
\frac{\theta}{2} \int_{B_{2 r}} v^{2}|\nabla u|^{2} d y \leq \frac{C}{\theta r^{2}} \int_{B_{2 r}}|u-\rho|^{2} d y+C|\sigma| \int_{B_{2 r}}|u|^{2} d y .
$$

Then, multiplying both sides by $\frac{2}{\theta}$ and using that $v=1$ on $B_{r}$ gives

$$
\int_{B_{r}}|\nabla u|^{2} d y \leq \frac{2 C}{\theta^{2} r^{2}} \int_{B_{2 r}}|u-\rho|^{2} d y+\frac{2 C|\sigma|}{\theta} \int_{B_{2 r}}|u|^{2} d y .
$$

Now, for the term $\int_{B_{2 r}}|u-\rho|^{2} d y$, we must consider two subcases.

Subcase 1.A. If $B_{2 r} \subset \Omega_{\varepsilon}$, then let

$$
\rho^{\alpha}=f_{B_{2 r}} u^{\alpha} d y
$$

Our condition on the support of $v$ implies $v^{2}(u-\rho) \in H_{0}^{1}\left(\Omega_{\varepsilon}\right)$. So, setting $q=2$ and $S=B_{2 r}$ in the Sobolev-Poincaré Inequality (3.2), we obtain

$$
\int_{B_{2 r}}|u-\rho|^{2} d y \leq C\left(\int_{B_{2 r}}|\nabla u|^{\frac{2 n}{n+2}} d y\right)^{\frac{n+2}{n}} .
$$

Using this estimate with (3.7) gives

$$
\int_{B_{r}}|\nabla u|^{2} d y \leq \frac{C}{r^{2}}\left(\int_{B_{2 r}}|\nabla u|^{\frac{2 n}{n+2}} d y\right)^{\frac{n+2}{n}}+C|\sigma| \int_{B_{2 r}}|u|^{2} d y .
$$

Now, dividing through by $r^{n}$ gives the desired result with $C_{3}=0$. 
Subcase 1.B. If $B_{2 r} \cap \Omega_{\varepsilon}^{c} \neq \emptyset$, then set $\rho=0$, which, again, guarantees that $v^{2}(u-\rho) \in H_{0}^{1}\left(\Omega_{\varepsilon}\right)$. So setting $q=2$ and $S=B_{4 r} \cap \Omega_{\varepsilon}$ in the Sobolev-Poincaré Inequality (3.2), we have by our assumption on $\Omega_{\varepsilon}^{c}(2.2)$ that

$$
\int_{B_{4 r}}|u-\rho|^{2} d y \leq C\left(\int_{B_{4 r}}|\nabla u|^{\frac{2 n}{n+2}} d y\right)^{\frac{n+2}{n}} .
$$

From (3.7), we obtain

$$
\int_{B_{r}}|\nabla u|^{2} d y \leq \frac{C}{r^{2}}\left(\int_{B_{4 r}}|\nabla u|^{\frac{2 n}{n+2}} d y\right)^{\frac{n+2}{n}}+C|\sigma| \int_{B_{4 r}}|u|^{2} d y .
$$

A simple covering argument gives the estimate with $B_{4 r}$ replaced with $B_{2 r}$.

Case 2: $\boldsymbol{L}$ satisfies the ellipticity condition (2.11). From (2.11) and (3.6), we have

$\int_{B_{r}} \tau|\kappa(u)|^{2} d y \leq \int_{B_{2 r}} \frac{\omega v^{2}|\nabla u|^{2}}{2} d y+\frac{C}{\omega r^{2}} \int_{B_{2 r}}|u-\rho|^{2} d y+C|\sigma| \int_{B_{2 r}}|u|^{2} d y$.

Also, by Korn's inequality (3.3), we have

$$
\frac{\tau}{C} \int_{B_{r}}|\nabla u|^{2} d y-\frac{\tau}{r^{2}} \int_{B_{r}}|u-\rho|^{2} d y \leq \int_{B_{r}} \tau|\kappa(u)|^{2} d y .
$$

This implies

$$
\begin{gathered}
\int_{B_{r}}|\nabla u|^{2} d y \leq \frac{C \omega}{2 \tau} \int_{B_{2 r}}|\nabla u|^{2} d y+C\left(\frac{1}{\omega \tau r^{2}}+\frac{1}{r^{2}}\right) \int_{B_{2 r}}|u-\rho|^{2} d y \\
+\frac{C|\sigma|}{\tau} \int_{B_{2 r}}|u|^{2} d y .
\end{gathered}
$$

This again leads to two subcases. We must choose $\rho$ appropriately and use the Sobolev-Poincaré inequality (3.2) as in case 1. Then, by taking $\omega$ sufficiently small, we obtain the desired result.

Case 3: $L$ satisfies the Legendre-Hadamard condition (2.4) with continuous coefficients in $\bar{\Omega}_{\varepsilon}$. We note that it suffices to study when $u \in C_{c}^{\infty}\left(\Omega_{\varepsilon}\right)$ and first consider when the coefficients are constant. We rewrite the left side of (3.5) as

$$
\begin{aligned}
& \int_{\Omega_{\varepsilon}} a_{i j}^{\alpha \beta}\left((u-\rho)^{\alpha} v\right)_{i}\left((u-\rho)^{\beta} v\right)_{j} d y \\
& \quad+\int_{\Omega_{\varepsilon}} a_{i j}^{\alpha \beta}\left[v v_{j} u_{i}^{\alpha}(u-\rho)^{\beta}-v_{i} v(u-\rho)^{\alpha} u_{j}^{\beta}-v_{i} v_{j}(u-\rho)^{\alpha}(u-\rho)^{\beta}\right] d y .
\end{aligned}
$$


This implies

$$
\begin{aligned}
& \int_{B_{2 r}} a_{i j}^{\alpha \beta}\left((u-\rho)^{\alpha} v\right)_{i}\left((u-\rho)^{\beta} v\right)_{j} d y \\
& \quad \leq C \int_{B_{2 r}}|\nabla v||\nabla((u-\rho) v)||u-\rho|+|u-\rho|^{2}|\nabla v|^{2}+|\sigma||u||u-\rho| d y .
\end{aligned}
$$

We note that we may use the Fourier transform to get a lower bound for the left side to achieve the estimate

$$
\int_{B_{2 r}}|\nabla((u-\rho) v)|^{2} d y \leq \frac{C}{r^{2}} \int_{B_{2 r}}|u-\rho|^{2} d y+C|\sigma| \int_{B_{2 r}}|u|^{2} d y .
$$

This implies the estimate

$$
\int_{B_{r}}|\nabla u|^{2} d y \leq \frac{C}{r^{2}} \int_{B_{2 r}}|u-\rho|^{2} d y+C|\sigma| \int_{B_{2 r}}|u|^{2} d y .
$$

So, again, if we employ the Sobolev-Poincaré inequality (3.2), we get the desired result in the case of constant coefficients. If the coefficients are continuous and nonconstant, then we freeze the coefficients at $x$. That is, from the weak formulation (2.3), we have

$$
\begin{aligned}
& \int_{\Omega_{\varepsilon}} a_{i j}^{\alpha \beta}(x) u_{i}^{\alpha}\left((u-\rho) v^{2}\right)_{j}^{\beta} d y+\int_{\Omega_{\varepsilon}}\left(a_{i j}^{\alpha \beta}-a_{i j}^{\alpha \beta}(x)\right) u_{i}^{\alpha}\left((u-\rho) v^{2}\right)_{j}^{\beta} d y \\
& \quad=\sigma \int_{\Omega_{\varepsilon}} u^{\gamma}\left((u-\rho) v^{2}\right)^{\gamma} d y .
\end{aligned}
$$

So, if we define the modulus of continuity to be

$$
M\left(x_{0}, R\right)=\max _{\substack{y \in \bar{B}_{R}\left(x_{0}\right) \\ i, j, \alpha, \beta}}\left|a_{i j}^{\alpha \beta}(y)-a_{i j}^{\alpha \beta}\left(x_{0}\right)\right|
$$

then we have that

$$
\begin{aligned}
\int_{B_{2 r}} & \left(a_{i j}^{\alpha \beta}-a_{i j}^{\alpha \beta}(x)\right) u_{i}^{\alpha}\left((u-\rho) v^{2}\right)_{j}^{\beta} d y \\
& \leq M(x, 2 r) \int_{B_{2 r}} v^{2}|\nabla u|^{2} d y+2 M(x, 2 r) \int_{B_{2 r}} v|\nabla v||\nabla u \| u-\rho| d y \\
& \leq C\left(M(x, 2 r)+M(x, 2 r)^{2}\right) \int_{B_{2 r}}|\nabla u|^{2} d y+\frac{C}{r^{2}} \int_{B_{2 r}}|u-\rho|^{2} d y .
\end{aligned}
$$

Also, by the uniform continuity of the coefficients on $\bar{\Omega}_{\varepsilon}$, for any $c<1$, there exists $r_{0}$ depending on $c$, so that if $C\left(x_{0}, R\right)=C\left(M\left(x_{0}, 2 R\right)+M\left(x_{0}, 2 R\right)^{2}\right)$ and $r \leq r_{0}$, then

$$
C\left(x_{0}, r\right) \leq c
$$


for all $x_{0} \in \bar{\Omega}_{\varepsilon}$. So, now moving the second term on the left side of (3.9) to the right and using the constant coefficient case (3.8), we obtain that for any $c<1$, there exists $r_{0}$ so that if $r \leq r_{0}$,

$$
\int_{B_{r}}|\nabla u|^{2} d y \leq \frac{C}{r^{2}} \int_{B_{2 r}}|u-\rho|^{2} d y+C|\sigma| \int_{B_{2 r}}|u|^{2} d y+c \int_{B_{2 r}}|\nabla u|^{2} d y .
$$

We again choose $\rho$ appropriately and apply the Sobolev-Poincaré inequality (3.2) to get the desired result.

As stated earlier, our proof of Theorem 2.1 relies on the gradient of an eigenfunction satisfying the reverse Hölder inequality, as in our next theorem.

Theorem 3.5. There exists $\varepsilon_{1}>0$ so that if $u$ is an eigenfunction with eigenvalue $\sigma$, then

$$
f_{\Omega_{\varepsilon}}|\nabla u|^{\tilde{p}} d y \leq C\left[\left(f_{\Omega_{\varepsilon}}|\nabla u|^{2} d y\right)^{\frac{\tilde{p}}{2}}+|\sigma|^{\frac{\tilde{p}}{2}} f_{\Omega_{\varepsilon}}|u|^{\tilde{p}} d y\right]
$$

where $2 \leq \tilde{p}<2+\varepsilon_{1}$, and $\varepsilon_{1}$ and $C$ are independent of $\varepsilon$ and any eigenvalue.

Proof. Now if $u$ is an eigenfunction with eigenvalue $\sigma$, we have $u \in H_{0}^{1}\left(\Omega_{\varepsilon}\right)$, and thus we may employ the Sobolev inequality to get that $|u| \in L^{r}\left(\Omega_{\varepsilon}\right)$ for some $r>2$. If $L$ satisfies either (2.6) or (2.11) with uniformly bounded coefficients, then we may choose a cube $Q_{R}$, centered at 0 , with sidelength $R$ such that $\Omega_{\varepsilon} \subset Q_{\frac{R}{2}}$, uniformly in $\varepsilon$, and set $g=|\nabla u|^{\frac{2 n}{n+2}}, f=\left(C_{3}|\sigma|\right)^{\frac{n}{n+2}}|u|^{\frac{2 n}{n+2}}, q=\frac{n+2}{n}$, and $u=0$ outside $\Omega_{\varepsilon}$, we may conclude by (3.4) and (3.1) that

$$
\left(f_{\Omega_{\varepsilon}}|\nabla u|^{\frac{2 n p}{n+2}} d y\right)^{\frac{1}{p}} \leq C\left[\left(f_{\Omega_{\varepsilon}}|\nabla u|^{2} d y\right)^{\frac{n}{n+2}}+|\sigma|^{\frac{n}{n+2}}\left(f_{\Omega_{\varepsilon}}|u|^{\frac{2 n p}{n+2}} d y\right)^{\frac{1}{p}}\right]
$$

where $\frac{n+2}{n} \leq p \leq \frac{n+2}{n}+\varepsilon$, which, from Theorem 3.4 is independent of $\varepsilon$ and any eigenvalue. So, setting $\tilde{p}=\frac{2 n p}{n+2}$, we have the result. If $L$ satisfies (2.4) with continuous coefficients, then since we only have Theorem 3.4 true for small $r$, we must cover $\Omega_{\varepsilon}$ with a fixed number of cubes and apply (3.1) to each cube to obtain the result.

\section{Stability of eigenvalues}

From this point, let $\sigma_{k}^{\varepsilon}$ be the $k$ th eigenvalue with respect to $\Omega_{\varepsilon}$, and $\varphi_{k}^{\varepsilon}$ be its corresponding eigenfunction with $\varphi_{k}^{\varepsilon}=0$ outside $\Omega_{\varepsilon}$ for $\varepsilon \geq 0$. We also fix an eigenvalue $\sigma_{J}^{0}$ with multiplicity $m_{J}$ where $\sigma_{J-1}^{0}<\sigma_{J}^{0}$ if $J \geq 2$. We will consider the family $\left\{\sigma_{J}^{\varepsilon}\right\}$ as $\varepsilon>0$ tends to 0 . We begin with the following proposition taken from Anné [2], pp. 2595-2596. 
Convergence of Dirichlet eigenvalues for elliptic systems on perturbed domains 305

Lemma 4.1. Let $(q, \mathcal{D})$ be a closed non-negative quadratic form with form domain $D$ in the Hilbert space $(\mathscr{H},\langle\cdot, \cdot\rangle)$. Define the associated norm $\|f\|_{1}^{2}=\|f\|_{\mathscr{H}}^{2}+q(f)$, and the spectral projector $\Pi_{I}$ for any interval $I=(\alpha, \beta)$ for which the boundary does not meet the spectrum.

(1) Suppose $f \in \mathscr{D}$ and $\lambda \in I$ satisfy

$$
|q(f, g)-\lambda\langle f, g\rangle| \leq \delta\|f\|\|g\|_{1}, \quad g \in \mathcal{D} .
$$

Then there exists a constant $C>0$, which depends on $I$, such that if a is less than the distance of $\alpha$ or $\beta$ to the spectrum of $q$,

$$
\left\|\Pi_{I}(f)-f\right\|_{1}=\left\|\Pi_{I^{c}}(f)\right\|_{1} \leq \frac{C \delta}{a}\|f\| .
$$

(2) Suppose the spectral space $E(I)$ has dimension $m$ and $f_{1}, \ldots, f_{m}$ is an orthonormal family which satisfies

$$
\left\|\Pi_{I^{c}}\left(f_{j}\right)\right\|_{1} \leq \delta, \quad j=1, \ldots, m .
$$

Also let $E$ be the space spanned by the $f_{j}$ 's. Then,

$$
\operatorname{dist}(E(I), E) \leq C \delta
$$

where the distance is measured as the distance between the two orthogonal projectors.

This lemma will give us the results we need for the convergence of eigenvalues. We will prove estimates on eigenfunctions using the reverse Hölder inequality (3.10), which will allow us to use this lemma. We start with the following proposition which follows immediately from the construction of eigenvalues.

Proposition 4.1. We have for any $\varepsilon>0$, and any $k \in \mathbb{N}$,

$$
\sigma_{k}^{\varepsilon} \leq \sigma_{k}^{0}
$$

This proposition gives us the easy half of the inequality in our theorem. To prove the second half of the inequality, we will need a few items.

Proposition 4.2. For any $\varepsilon>0$, and $k \geq 1$, if $\varphi=\varphi_{k}^{\varepsilon}$, then we have

$$
\int_{\Omega_{\varepsilon}}|\nabla \varphi|^{\tilde{p}} d y \leq C
$$

where $\tilde{p}>2$ is from (3.10), and $C$ depends on $\left|\Omega_{0}\right|$ and $n$, with order, for $n \geq 3$, $\mathcal{O}\left(\left|\sigma_{k}^{0}\right|^{\frac{2 \tilde{p}+n(\tilde{p}-2)}{4}}\right)$ or, for $n=2, \mathcal{O}\left(\left|\sigma_{k}^{0}\right|^{\frac{q \tilde{p}+2(\tilde{p}-q)}{2 q}}\right)$, where $2-\xi<q<2$ for small $\xi$. Furthermore, $\tilde{p}$ and $C$ are independent of $\varepsilon$ and if $n=2, C$ blows up as $q \rightarrow 2$. 
Proof. Now, from (3.10), we have

$$
\int_{\Omega_{\varepsilon}}|\nabla \varphi|^{\tilde{p}} d y \leq C\left[\left|\Omega_{\varepsilon}\right|^{\frac{2-\tilde{p}}{2}}\left(\int_{\Omega_{\varepsilon}}|\nabla \varphi|^{2} d y\right)^{\frac{\tilde{p}}{2}}+\left|\sigma_{k}^{\varepsilon}\right|^{\frac{\tilde{p}}{2}}\left(\int_{\Omega_{\varepsilon}}|\varphi|^{\tilde{p}} d y\right)\right]
$$

where $\tilde{p}>2$ is from (3.10). Recall that by Gårding's inequality (2.5) and since $\varphi$ is an eigenfunction, we have

$$
\begin{aligned}
C_{1} \int_{\Omega_{\varepsilon}}|\nabla \varphi|^{2} d y & \leq \int_{\Omega_{\varepsilon}} a_{i j}^{\alpha \beta} \varphi_{i}^{\alpha} \varphi_{j}^{\beta} d y+C_{2} \int_{\Omega_{\varepsilon}}|\varphi|^{2} d y \\
& \leq C\left(1+\left|\sigma_{k}^{\varepsilon}\right|\right) \int_{\Omega_{\varepsilon}}|\varphi|^{2} d y \\
& \leq C\left(1+\left|\sigma_{k}^{\varepsilon}\right|\right)
\end{aligned}
$$

the last line owing to the normalization of the eigenfunctions. Next, we will consider $n \geq 3$ and estimate

$$
\int_{\Omega_{\varepsilon}}|\varphi|^{\tilde{p}} d y
$$

Using Sobolev's inequality and (4.4), we have

$$
\left(\int_{\Omega_{\varepsilon}}|\varphi|^{\frac{2 n}{n-2}} d y\right)^{\frac{n-2}{2 n}} \leq C\left(\int_{\Omega_{\varepsilon}}|\nabla \varphi|^{2} d y\right)^{\frac{1}{2}} \leq C\left(1+\left|\sigma_{k}^{\varepsilon}\right|^{\frac{1}{2}}\right) .
$$

Also, by Hölder's inequality, we have

$$
\left(\int_{\Omega_{\varepsilon}}|\varphi|^{\tilde{p}} d y\right)^{\frac{1}{\tilde{p}}} \leq\left(\int_{\Omega_{\varepsilon}}|\varphi|^{2} d y\right)^{\frac{1-t}{2}}\left(\int_{\Omega_{\varepsilon}}|\varphi|^{\frac{2 n}{n-2}} d y\right)^{\frac{t(n-2)}{2 n}}
$$

where $t$ satisfies

$$
\frac{1}{\tilde{p}}=\frac{1-t}{2}+\frac{t(n-2)}{2 n} .
$$

From this inequality and (4.4), it follows that

$$
\left(\int_{\Omega_{\varepsilon}}|\varphi|^{\tilde{p}} d y\right)^{\frac{1}{\tilde{p}}} \leq C\left(1+\left|\sigma_{k}^{\varepsilon}\right|^{\frac{t}{2}}\right)=C\left(1+\left|\sigma_{k}^{\varepsilon}\right|^{\frac{n(\tilde{p}-2)}{4 \tilde{p}}}\right) .
$$

Now, using this inequality along with (4.3), (4.4), and (4.1), we obtain

$$
\begin{aligned}
\int_{\Omega_{\varepsilon}}|\nabla \varphi|^{\tilde{p}} d y & \leq C\left[\left(1+\left|\sigma_{k}^{0}\right|\right)^{\frac{\tilde{p}}{2}}+\left|\sigma_{k}^{0}\right|^{\frac{\tilde{p}}{2}}\left(1+\left|\sigma_{k}^{0}\right|^{\frac{n(\tilde{p}-2)}{4}}\right)\right] \\
& \leq C\left[\left|\sigma_{k}^{0}\right|^{\frac{2 \tilde{p}+n(\tilde{p}-2)}{4}}+\left|\sigma_{k}^{0}\right|^{\frac{\tilde{p}}{2}}+1\right] .
\end{aligned}
$$

This completes the proof for $n \geq 3$. 
Convergence of Dirichlet eigenvalues for elliptic systems on perturbed domains 307 If $n=2$, then from Sobolev's inequality, Hölder's inequality, and (4.4), we have

$$
\begin{aligned}
\left(\int_{\Omega_{\varepsilon}}|\varphi|^{q^{*}} d y\right)^{\frac{1}{q^{*}}} & \leq \frac{C}{(2-q)^{\frac{1}{2}}}\left(\int_{\Omega_{\varepsilon}}|\nabla \varphi|^{q} d y\right)^{\frac{1}{q}} \\
& \leq \frac{C}{(2-q)^{\frac{1}{2}}}\left(\int_{\Omega_{\varepsilon}}|\nabla \varphi|^{2} d y\right)^{\frac{1}{2}}\left|\Omega_{\varepsilon}\right|^{\frac{1}{q^{*}}} \\
& \leq \frac{C}{(2-q)^{\frac{1}{2}}}\left(1+\left|\sigma_{k}^{\varepsilon}\right|^{\frac{1}{2}}\right)
\end{aligned}
$$

where $q^{*}=\frac{2 q}{q-2}$ is the Sobolev conjugate of $q$. Then, again applying Hölder's inequality, we obtain

$$
\left(\int_{\Omega_{\varepsilon}}|\varphi|^{\tilde{p}} d y\right)^{\frac{1}{\tilde{p}}} \leq \frac{C}{(2-q)^{\frac{t}{2}}}\left(1+\left|\sigma_{k}^{\varepsilon}\right|^{\frac{t}{2}}\right)=\frac{C}{(2-q)^{\frac{(\tilde{p}-q)}{\tilde{p} q}}}\left(1+\left|\sigma_{k}^{\varepsilon}\right|^{\frac{(\tilde{p}-q)}{\tilde{p} q}}\right) .
$$

Now using (4.3), (4.4), and (4.1), we obtain

$$
\begin{aligned}
\int_{\Omega_{\varepsilon}}|\nabla \varphi|^{\tilde{p}} d y & \leq \frac{C}{(2-q)^{\frac{(\tilde{p}-q)}{q}}}\left[\left(1+\left|\sigma_{k}^{0}\right|\right)^{\frac{\tilde{p}}{2}}+\left|\sigma_{k}^{0}\right|^{\frac{\tilde{p}}{2}}\left(1+\left|\sigma_{k}^{0}\right|^{\frac{(\tilde{p}-q)}{q}}\right)\right] \\
& \leq \frac{C}{(2-q)^{\frac{(\tilde{p}-q)}{q}}}\left[\left|\sigma_{k}^{0}\right|^{\frac{q \tilde{p}+2(\tilde{p}-q)}{2 q}}+\left|\sigma_{k}^{0}\right|^{\frac{\tilde{p}}{2}}+1\right] .
\end{aligned}
$$

Lemma 4.2. For the eigenfunction $\varphi_{k}^{\varepsilon}, J \leq k \leq J+m_{J}-1$, and any $w \in H_{0}^{1}\left(\Omega_{0}\right)$, we have the following estimate:

$$
\left|\int_{\Omega_{0}} a_{i j}^{\alpha \beta}\left(\eta_{\varepsilon} \varphi_{k}^{\varepsilon}\right)_{i}^{\alpha} w_{j}^{\beta} d y-\sigma_{k}^{\varepsilon} \int_{\Omega_{0}}\left(\eta_{\varepsilon} \varphi_{k}^{\varepsilon}\right)^{\alpha} w^{\alpha} d y\right| \leq C \varepsilon^{\frac{n(\tilde{p}-2)}{2 \tilde{p}}}\|w\|_{1}
$$

where $\|w\|_{1}$ is from Lemma 4.1 with

$$
q(f, g)=\int_{\Omega_{0}} a_{i j}^{\alpha \beta} f_{i}^{\alpha} g_{j}^{\beta} d y
$$

and $C$ only depends on $\left|\Omega_{0}\right|, n, \sigma_{J}^{0}$, and is independent of $\varepsilon$. 
Proof. First, recall that $w$ is extended to be 0 outside $\Omega_{0}$ and $\varphi_{k}^{\varepsilon}$ is extended to be 0 in $\left(B_{\varepsilon} \cup \tilde{B}_{\varepsilon}\right) \cap \Omega_{\varepsilon}^{c}$. We have

$$
\begin{aligned}
& \left|\int_{\Omega_{0}} a_{i j}^{\alpha \beta}\left(\eta_{\varepsilon} \varphi_{k}^{\varepsilon}\right)_{i}^{\alpha} w_{j}^{\beta} d y-\sigma_{k}^{\varepsilon} \int_{\Omega_{0}}\left(\eta_{\varepsilon} \varphi_{k}^{\varepsilon}\right)^{\alpha} w^{\alpha} d y\right| \\
& \leq\left|\int_{\Omega_{0}} a_{i j}^{\alpha \beta}\left[\left(\eta_{\varepsilon}\right)_{i}\left(\varphi_{k}^{\varepsilon}\right)^{\alpha} w_{j}^{\beta}-\left(\eta_{\varepsilon}\right)_{j}\left(\varphi_{k}^{\varepsilon}\right)^{\alpha} w^{\beta}\right] d y\right| \\
& \quad+\left|\int_{\Omega_{\varepsilon}} a_{i j}^{\alpha \beta}\left(\varphi_{k}^{\varepsilon}\right)_{i}^{\alpha}\left(\eta_{\varepsilon} w\right)_{j}^{\beta} d y-\sigma_{k}^{\varepsilon} \int_{\Omega_{\varepsilon}}\left(\varphi_{k}^{\varepsilon}\right)^{\alpha}\left(\eta_{\varepsilon} w\right)^{\alpha} d y\right| \\
& =|\mathrm{I}+\mathrm{II}|+|\mathrm{III}+\mathrm{IV}| .
\end{aligned}
$$

First, since $\varphi_{k}^{\varepsilon}$ is an eigenfunction with eigenvalue $\sigma_{k}^{\varepsilon}$, we have that III + IV $=0$. Also, by Hölder's inequality and Poincaré's inequality, we have

$$
\begin{aligned}
|\mathrm{I}+\mathrm{II}| & \leq \frac{C}{\varepsilon}\left\|\varphi_{k}^{\varepsilon}\right\|_{L^{2}\left(\boldsymbol{B}_{\varepsilon} \cup \tilde{\boldsymbol{B}}_{\varepsilon}\right)}\left(\|\nabla w\|_{L^{2}\left(\boldsymbol{B}_{\varepsilon} \cup \tilde{\left.\boldsymbol{B}_{\varepsilon}\right)}\right.}+\|w\|_{L^{2}\left(\boldsymbol{B}_{\varepsilon} \cup \tilde{\boldsymbol{B}_{\varepsilon}}\right)}\right) \\
& \leq C\left\|\nabla \varphi_{k}^{\varepsilon}\right\|_{L^{2}\left(\boldsymbol{B}_{\varepsilon} \cup \tilde{\boldsymbol{B}}_{\varepsilon}\right)}\|w\|_{1}
\end{aligned}
$$

where we have used Gårding's inequality (2.5) on the last line for $w$. Thus, from Hölder's inequality and Proposition 4.2,

$$
\begin{aligned}
|\mathrm{I}+\mathrm{II}| & \leq C \varepsilon^{\frac{n(\tilde{p}-2)}{2 \tilde{p}}}\left\|\nabla \varphi_{k}^{\varepsilon}\right\|_{L^{\tilde{p}}\left(\Omega_{\varepsilon}\right)}\|w\|_{1} \\
& \leq C \varepsilon^{\frac{n(\tilde{p}-2)}{2 \tilde{p}}}\|w\|_{1} .
\end{aligned}
$$

Since $\sigma_{k}^{0}=\sigma_{J}^{0}$, the proof of the lemma is concluded.

If we choose an interval $I$ around $\sigma_{k}^{0}$ such that $\sigma_{k}^{\varepsilon} \in I$, and let

$$
q(f, g)=\int_{\Omega_{0}} a_{i j}^{\alpha \beta} f_{i}^{\alpha} g_{j}^{\beta} d y
$$

and $f=\eta_{\varepsilon} \varphi_{k}^{\varepsilon}$, we aim to satisfy the hypotheses for Part 1 of Lemma 4.1. In order to do this, we need $\left\|\eta_{\varepsilon} \varphi_{k}^{\varepsilon}\right\|_{L^{2}\left(\Omega_{0}\right)}$ to be bounded away from 0 . To achieve this, we start with the following well-known proposition.

Proposition 4.3. If $A$ is an $N \times N$ matrix and $v$ is a $N \times 1$ vector such that $A v=0$ and

$$
\sum_{i \neq l}^{N}\left|A_{l i}\right|<\left|A_{l l}\right|, \quad l=1, \ldots, N,
$$

then $v=0$. 
Convergence of Dirichlet eigenvalues for elliptic systems on perturbed domains 309

The next proposition shows that the functions $\left\{\eta_{\varepsilon} \varphi_{k}^{\varepsilon}\right\}_{k=J}^{J+m_{J}-1}$ are almost orthonormal.

Proposition 4.4. For any $\varepsilon>0$ and $l, k \in \mathbb{N},\left(J \leq l, k \leq J+m_{J}-1\right)$, if $\varphi_{k}=\varphi_{k}^{\varepsilon}$, we have the following estimates:

$$
\int_{\Omega_{\varepsilon}} \eta_{\varepsilon}^{2}\left|\varphi_{k}\right|^{2} d y \geq 1-C \varepsilon^{\frac{d(\tilde{p}-2)}{\tilde{p}}}
$$

and

$$
\left|\int_{\Omega_{\varepsilon}} \eta_{\varepsilon}^{2} \varphi_{k} \cdot \varphi_{l} d y\right| \leq C \varepsilon^{\frac{d(\tilde{p}-2)}{\tilde{p}}} \text { if } k \neq l \text {, }
$$

where $C$ only depends on $\left|\Omega_{0}\right|, n$, and $\sigma_{J}^{0}$, and is independent of $\varepsilon$.

Proof. We start by showing (4.6). Since the eigenfunctions are normalized, we obtain for each $k$,

$$
\begin{aligned}
1-\int_{\Omega_{\varepsilon}} \eta_{\varepsilon}^{2}\left|\varphi_{k}\right|^{2} d y & =\int_{\Omega_{\varepsilon}}\left(1-\eta_{\varepsilon}^{2}\right)\left|\varphi_{k}\right|^{2} d y \\
& =\int_{T_{\varepsilon} \cup B_{\varepsilon} \cup \tilde{B}_{\varepsilon}}\left(1-\eta_{\varepsilon}^{2}\right)\left|\varphi_{k}\right|^{2} d y \\
& \leq\left\|\nabla_{k}\right\|_{L^{\tilde{p}}\left(\Omega_{\varepsilon}\right)}^{2}\left|T_{\varepsilon} \cup B_{\varepsilon} \cup \tilde{B}_{\varepsilon}\right|^{\frac{\tilde{p}-2}{\tilde{p}}} \\
& \leq C_{k} \frac{d(\tilde{p}-2)}{\tilde{p}}
\end{aligned}
$$

where, from (4.2), $C_{k}$ depends on $\sigma_{k}^{0}$. Again, since $\sigma_{k}^{0}=\sigma_{J}^{0}$, we have (4.6).

Next, to show (4.7), we have

$$
\begin{aligned}
& \left|\int_{\Omega_{\varepsilon}} \eta_{\varepsilon}^{2} \varphi_{k} \cdot \varphi_{l} d y\right| \\
& \quad \leq\left|\int_{B_{\varepsilon} \cup \widetilde{B}_{\varepsilon}} \eta_{\varepsilon}^{2} \varphi_{k} \cdot \varphi_{l} d y\right|+\left|\int_{\Omega_{0} \backslash\left(B_{\varepsilon} \cup \widetilde{B}_{\varepsilon}\right)} \eta_{\varepsilon}^{2} \varphi_{k} \cdot \varphi_{l} d y\right| \\
& \quad=\left|\int_{B_{\varepsilon} \cup \widetilde{B}_{\varepsilon}} \eta_{\varepsilon}^{2} \varphi_{k} \cdot \varphi_{l} d y\right|+\left|\int_{\Omega_{0} \backslash\left(B_{\varepsilon} \cup \widetilde{B}_{\varepsilon}\right)} \varphi_{k} \cdot \varphi_{l} d y-\int_{\Omega_{\varepsilon}} \varphi_{k} \cdot \varphi_{l} d y\right| \\
& \quad \leq \int_{B_{\varepsilon} \cup \widetilde{B}_{\varepsilon}}\left|\varphi_{k} \cdot \varphi_{l}\right| d y+\int_{T_{\varepsilon} \cup B_{\varepsilon} \cup \widetilde{B}_{\varepsilon}}\left|\varphi_{k} \cdot \varphi_{l}\right| d y
\end{aligned}
$$


the second inequality following since the set of eigenfunctions form an orthogonal set in $L^{2}\left(\Omega_{\varepsilon}\right)$. So, next by Hölder's inequality, we get

$$
\begin{aligned}
\left|\int_{\Omega_{\varepsilon}} \eta_{\varepsilon}^{2} \varphi_{k} \cdot \varphi_{l} d y\right| \leq & \left(\int_{B_{\varepsilon} \cup \widetilde{\boldsymbol{B}}_{\varepsilon}}\left|\varphi_{k}\right|^{2} d y\right)^{\frac{1}{2}}\left(\int_{B_{\varepsilon} \cup \widetilde{B}_{\varepsilon}}\left|\varphi_{l}\right|^{2} d y\right)^{\frac{1}{2}} \\
& +\left(\int_{T_{\varepsilon} \cup B_{\varepsilon} \cup \widetilde{B}_{\varepsilon}}\left|\varphi_{k}\right|^{2} d y\right)^{\frac{1}{2}}\left(\int_{T_{\varepsilon} \cup B_{\varepsilon} \cup \widetilde{B}_{\varepsilon}}\left|\varphi_{l}\right|^{2} d y\right)^{\frac{1}{2}} \\
= & \text { I }+ \text { II. }
\end{aligned}
$$

Now, from Poincaré's inequality and (4.2), we get

$$
\begin{aligned}
& \mathrm{I} \leq\left[\left(\int_{\boldsymbol{B}_{\varepsilon} \cup \widetilde{\boldsymbol{B}}_{\varepsilon}}\left|\varphi_{k}\right|^{\tilde{p}} d y\right)^{\frac{2}{\tilde{p}}}\left|B_{\varepsilon} \cup \tilde{B}_{\varepsilon}\right|^{\frac{\tilde{p}-2}{\tilde{p}}}\right]^{\frac{1}{2}}\left[\left(\int_{B_{\varepsilon} \cup \tilde{\boldsymbol{B}}_{\varepsilon}}\left|\varphi_{l}\right|^{\tilde{p}} d y\right)^{\frac{2}{\tilde{p}}}\left|B_{\varepsilon} \cup \tilde{B}_{\varepsilon}\right|^{\frac{\tilde{p}-2}{\tilde{p}}}\right]^{\frac{1}{2}}
\end{aligned}
$$

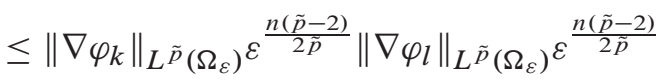

$$
\begin{aligned}
& \leq C_{k} \varepsilon^{\frac{n(\tilde{p}-2)}{2 \tilde{p}}} C_{l} \varepsilon^{\frac{n(\tilde{p}-2)}{2 \tilde{p}}}
\end{aligned}
$$

where $C_{k}$ again depends on $\sigma_{k}^{0}$ and $C_{l}$ depends on $\sigma_{l}^{0}$. Thus, we have

$$
\mathrm{I} \leq C \varepsilon^{\frac{n(\tilde{p}-2)}{\tilde{p}}}
$$

where $C$ depends only on $\left|\Omega_{0}\right|, n$, and $\sigma_{J}^{0}$. Similarly,

$$
\mathrm{II} \leq C \varepsilon^{\frac{d(\tilde{p}-2)}{\tilde{p}}},
$$

so that the proposition is proved.

Note that with the aid of Lemma 4.2 and Proposition 4.4, if $\varepsilon$ is small enough, we have satisfied the hypotheses for Part 1 of Lemma 4.1 with

$$
q(f, g)=\int_{\Omega_{0}} a_{i j}^{\alpha \beta} f_{i}^{\alpha} g_{j}^{\beta} d y
$$

and $f=\eta_{\varepsilon} \varphi_{k}^{\varepsilon}$. Here, we relabel $\varepsilon_{1}$ to be small enough to achieve this for any $\varepsilon \leq \varepsilon_{1}$, and note that $\varepsilon_{1}$ only depends on fixed parameters. To satisfy the hypotheses for Part 2 of Lemma 4.1, we need an orthonormal basis. The next proposition shows that for small $\varepsilon$, we have a basis.

Proposition 4.5. The $\operatorname{set}\left\{\eta_{\varepsilon} \varphi_{k}^{\varepsilon}\right\}_{k=J}^{N}$ forms a linearly independent set for any $N \geq J$, for $0<\varepsilon \leq \varepsilon_{0}(N)$, where $\varepsilon_{0}(N)$ depends on $N$. 
Proof. Assume $C_{J} \eta_{\varepsilon} \varphi_{J}^{\varepsilon}+\cdots+C_{N} \eta_{\varepsilon} \varphi_{N}^{\varepsilon}=0$. Then, multiplying this equation by $\eta_{\varepsilon} \varphi_{l}^{\varepsilon}$, we obtain

$$
\sum_{k=J}^{N} C_{k}\left\langle\eta_{\varepsilon} \varphi_{k}^{\varepsilon}, \eta_{\varepsilon} \varphi_{l}^{\varepsilon}\right\rangle_{L^{2}\left(\Omega_{\varepsilon}\right)}=0, \quad l=J, \ldots, N
$$

So, if $A_{k l}=\left\langle\eta_{\varepsilon} \varphi_{k}^{\varepsilon}, \eta_{\varepsilon} \varphi_{l}^{\varepsilon}\right\rangle_{L^{2}\left(\Omega_{\varepsilon}\right)}$, we obtain by (4.6) and (4.7) that

$$
\left|A_{k k}\right| \geq 1-C \varepsilon^{\frac{d(\tilde{p}-2)}{\tilde{p}}}>C \varepsilon^{\frac{d(\tilde{p}-2)}{\tilde{p}}} \geq \sum_{\substack{k=J \\ k \neq l}}^{N}\left|A_{k l}\right|
$$

if $\varepsilon \leq \varepsilon(N)$, where $\varepsilon(N)$ depends on $N$ due to applying (4.7) $N-J$ times. Thus, we may use Proposition 4.3 to see that by setting $C=\left(C_{J}, \ldots, C_{N}\right)^{t}$, we have $C=0$, so that the proposition is proved.

Now we define

$$
J_{0}: L^{2}\left(\Omega_{\varepsilon}\right) \longrightarrow L^{2}\left(\Omega_{0}\right)
$$

to be given by

$$
J_{0} f=\eta_{\varepsilon} f
$$

and, similarly, we define

$$
J_{\varepsilon}: L^{2}\left(\Omega_{0}\right) \longrightarrow L^{2}\left(\Omega_{\varepsilon}\right)
$$

to be such that

$$
J_{\varepsilon} f(x)= \begin{cases}f(x) & \text { if } x \in \Omega_{0}, \\ 0 & \text { if } x \in \Omega_{\varepsilon} \backslash \Omega_{0}\end{cases}
$$

Let

$$
I=\left(\sigma_{J}^{0}-M \varepsilon^{\frac{n(\tilde{p}-2)}{4 \tilde{p}}}, \frac{\sigma_{J}^{0}+\sigma_{J+m_{J}}^{0}}{2}\right)
$$

for $M>0$ to be chosen later. Also, let $\Pi$ be the projector onto the space spanned by the eigenfunctions corresponding to the eigenvalues, $\left\{\sigma_{k}^{\varepsilon}\right\}_{k=J}^{N}$, in $I$. We first consider $\varepsilon=\varepsilon_{1}$. By Proposition 4.1, we may choose $M=M\left(\varepsilon_{1}\right)$ so that $\sigma_{k}^{\varepsilon}$ is in $I$ for $J \leq k \leq N$, where $N \geq J+m_{J}-1$, and where $N$ depends on $\varepsilon_{1}$. We next note that as $\varepsilon$ gets smaller, we may choose $M=M(\varepsilon)$ so that the set of eigenvalues in $I,\left\{\sigma_{k}^{\varepsilon}\right\}_{k=J}^{N_{0}}$, will have index $N_{0}$ in the range $J+m_{J}-1 \leq N_{0} \leq N$ since our family $\left\{\Omega_{\varepsilon}\right\}$ is nested. Our aim is to show that for $\varepsilon$ small, $N_{0}=J+m_{J}-1$.

We apply Proposition 4.5 to get the existence of $\varepsilon_{0}(N) \leq \varepsilon_{1}$ so that $\left\{\eta_{\varepsilon} \varphi_{k}^{\varepsilon}\right\}_{k=J}^{N_{0}}$ is a linearly independent set for $\varepsilon \leq \varepsilon_{0}(N)$ and for any $N_{0}$ in the range $J+m_{J}-1 \leq$ $N_{0} \leq N$. Then, we choose $M=M(\varepsilon(N))$ so that $\left\{\eta_{\varepsilon} \varphi_{k}^{\varepsilon}\right\}_{k=J}^{N_{0}}$ is also a basis for the 
range of $J_{0} \Pi J_{\varepsilon}$. Thus, we may apply the Gram-Schmidt process to this basis. That is, define

$$
\begin{aligned}
f_{J} & =\eta_{\varepsilon} \varphi_{J}^{\varepsilon}, \\
& \vdots \\
f_{k} & =\eta_{\varepsilon} \varphi_{k}^{\varepsilon}-\frac{\left\langle\eta_{\varepsilon} \varphi_{k}^{\varepsilon}, f_{J}\right\rangle}{\left\|f_{J}\right\|^{2}} f_{J}-\cdots-\frac{\left\langle\eta_{\varepsilon} \varphi_{k}^{\varepsilon}, f_{k-1}\right\rangle}{\left\|f_{k-1}\right\|^{2}} f_{k-1},
\end{aligned}
$$

We have the following lemma.

Lemma 4.3. Let I be as defined above. For each $k, J \leq k \leq J+m_{J}-1$, we have

$$
\left\|\Pi_{I^{c}}\left(f_{k}\right)\right\|_{1} \leq \frac{C \varepsilon^{\frac{d(\tilde{p}-2)}{4 \tilde{p}}}}{M},
$$

for $\varepsilon \leq \varepsilon(N)$, and where $M$ only depends on $\sigma_{J}^{0}, \sigma_{J-1}^{0}$, and $\varepsilon(N)$.

Proof. Following the previous arguments, when $\varepsilon=\varepsilon(N)$, we find $M=M(\varepsilon(N))$ so that $\left\{\eta_{\varepsilon} \varphi_{k}^{\varepsilon}\right\}_{k=J}^{N_{0}}$ is a basis for the range of $J_{0} \Pi J_{\varepsilon}$, and then apply the GramSchmidt process to this basis. We note the dependence on $\sigma_{J}^{0}$ and $\sigma_{J-1}^{0}$ is so that we only have 1 eigenvalue (with respect to $\Omega_{0}$ ) in $I$. So, defining

$$
q(f, g)=\int_{\Omega_{0}} a_{i j}^{\alpha \beta} f_{i}^{\alpha} g_{j}^{\beta} d y,
$$

we may apply Lemma 4.2, Proposition 4.4, and then Lemma 4.1 (Part 1) to obtain

$$
\left\|\Pi_{I^{c}}\left(f_{J}\right)\right\|_{1} \leq \frac{C \varepsilon(N)^{\frac{d(\tilde{p}-2)}{4 \tilde{p}}}}{M(\varepsilon(N))}
$$

where $C$ depends on $\left|\Omega_{0}\right|, n, \sigma_{J}^{0}$, and $\sigma_{J+m_{J}}^{0}$. Then, from Proposition 4.4, Lemma 4.2, and properties of the norm, we get the result for $\varepsilon(N)$ and $J \leq k \leq J+m_{J}-1$. Then, for $\varepsilon \leq \varepsilon(N)$, we may repeat this argument to get the result with $\varepsilon(N)$ replaced with $\varepsilon$ and $M(\varepsilon(N)$ replaced with $M(\varepsilon)$. But, since $M(\varepsilon(N)) \leq M(\varepsilon)$, we obtain the desired result for $\varepsilon \leq \varepsilon(N)$.

We now let $E=\operatorname{span}\left\{\varphi_{k}^{\varepsilon}\right\}_{k=J}^{J+m_{J}-1}$. Also, let $\Pi_{I}$ be the spectral projector corresponding to the eigenvalue $\sigma_{J}^{0}$ and $\Pi_{E}$ be the spectral projector onto $E$.

Corollary 4.1. We have

$$
\left\|\Pi_{I}-J_{0} \Pi_{E} J_{\varepsilon}\right\|_{\mathscr{L}\left\{L^{2}\left(\Omega_{0}\right)\right\}} \leq \frac{C \varepsilon^{\frac{d(\tilde{p}-2)}{4 \tilde{p}}}}{M},
$$


for $\varepsilon \leq \varepsilon(N)$, where $M$ only depends on $\sigma_{J}^{0}, \sigma_{J-1}^{0}$, and $\varepsilon(N)$. Consequently, for some $\varepsilon(J), N_{0}=J+m_{J}-1$ when $\varepsilon \leq \varepsilon(J)$.

Proof. Again, we first show for $\varepsilon=\varepsilon(N)$. Normalize the $f_{k}$ 's and observe that

$$
\frac{1}{\left\|f_{k}\right\|} \leq \frac{1}{1-C \varepsilon(N)^{\frac{d(\tilde{p}-2)}{2 \tilde{p}}}}
$$

Then apply Lemma 4.1 (Part 2) to the normalized functions. Then for general $\varepsilon \leq$ $\varepsilon(N)$, we note that since Lemma 4.3 is true with a uniform $M$, we obtain

$$
\left\|\Pi_{I}-J_{0} \Pi_{E} J_{\varepsilon}\right\|_{\mathscr{L}\left\{L^{2}\left(\Omega_{0}\right)\right\}} \leq \frac{C \varepsilon^{\frac{d(\tilde{p}-2)}{4 \tilde{p}}}}{M} .
$$

We next note that if $N_{0}>J+m_{J}-1$ for all $\varepsilon \leq \varepsilon(N)$, then we may find another projector $\Pi_{A}$ so that

$$
\left\|\Pi_{I}-J_{0} \Pi_{A} J_{\varepsilon}\right\|_{\mathscr{L}\left\{L^{2}\left(\Omega_{0}\right)\right\}} \leq \frac{C \varepsilon^{\frac{d(\tilde{p}-2)}{4 \tilde{p}}}}{M} .
$$

But this would mean

$$
\left\|J_{0} \Pi_{E} J_{\varepsilon}-J_{0} \Pi_{A} J_{\varepsilon}\right\|_{\mathscr{L}\left\{L^{2}\left(\Omega_{0}\right)\right\}} \leq \frac{C \varepsilon^{\frac{d(\tilde{p}-2)}{4 \tilde{p}}}}{M} .
$$

Therefore, for some $\varepsilon(J), N_{0}=J+m_{J}-1$, when $\varepsilon \leq \varepsilon(J)$.

Proof of Theorem 2.1. We first prove for $J=1$. By Corollary 4.1, for $\varepsilon \leq \varepsilon(1)$, we obtain $m_{1}=N_{0}$. This implies that $\left|\sigma_{k}^{\varepsilon}-\sigma_{k}^{0}\right| \leq C \varepsilon^{\frac{d(\tilde{p}-2)}{4 \tilde{p}}}$ only for $k, 1 \leq k \leq m_{1}$, and hence, the result for $J=1$. The result for $J=1$ implies that not only may we choose $M$ so that all eigenvalues $\left\{\sigma_{k}^{\varepsilon}\right\}_{k=m_{1}+1}^{m_{1}+m_{2}}$ are in the interval corresponding to the next highest eigenvalue $\sigma_{m_{1}+1}^{0}$, but also that $\sigma_{1}^{0}$ is not in this interval. Thus, we apply the same reasoning here to get the result for $\sigma_{m_{1}+1}^{0}$. Then, by an induction argument, we get the result for each $J \in \mathbb{N}$, satisfying $\sigma_{J}^{0}>\sigma_{J-1}^{0}$. We note here that since $\mathrm{C}$ depends on $\varepsilon(J)$, it depends on the multiplicity $J$.

We note that this paper introduces the use of $L^{p}$-estimates obtained by the reverse Hölder technique to the study of spectral problems for elliptic operators. Thus, this technique may be useful in studying spectral problems in situations where we do not know if higher regularity of solutions is true. We close by listing some open problems.

- If we have some additional regularity on the domain, can we use the methods from this work to get convergence of Neumann eigenvalues for general elliptic systems? 
- For elliptic systems on a symmetric dumbbell region with a straight tube, can we achieve upper and lower bounds on the splitting between the smallest eigenvalues?

- Can we investigate this problem further to see if a better rate of convergence exists?

\section{References}

[1] C. Anné and B. Colbois, Spectre du Laplacien agissant sur les $p$-formes différentielles et écrasement d'anses. Math. Ann. 303 (1995), 545-573. MR 1355003 Zbl 0909.58054

[2] C. Anné, A note on the generalized Dumbbell problem. Proc. Amer. Math. Soc. 123 (1995), 2595-2599. MR 1257096 Zbl 0829.58044

[3] J. M. Arrieta. Rates of eigenvalues on a Dumbbell domain. Simple eigenvalue case. Trans. Amer. Math. Soc. 347 (1995)), 3503-3531. MR 1297521 Zbl 0856.35095

[4] J. M. Arrieta, J. K. Hale, and Q. Han, Eigenvalue problems for nonsmoothly perturbed domains. J. Differential Equations 91 (1991), 24-52. MR 1106116 Zbl 0736.35073

[5] I. Babuska and R. Výborný, Continuous dependence of eigenvalues on the domain. Czechoslovak Math. J. 15 (1995), 169-178. MR 0182799 Zbl 0137.32302

[6] R. M. Brown, P. D. Hislop, and A. Martinez, Lower bounds on the interaction between cavities connected by a thin tube. Duke Math. J. 73 (1994), 163-176. MR 1257281 Zbl 0802.35109

[7] R. M. Brown, P. D. Hislop, and A. Martinez, Eigenvalues and resonances for domains with tubes: Neumann boundary conditions. J. Differential Equations 115 (1995), 458476. MR 1310941 Zbl 0815.35075

[8] V. I. Burenkov and P. D. Lamberti, Sharp spectral stability estimates via the Lebesgue measure of domains for higher order elliptic operators. Preprint 2010. arXiv:1012.4961v1

[9] I. Chavel and E. A. Feldman, Spectra of manifolds with small handles. Comment. Math. Helv. 56 (1981), 83-102. MR 0615617 Zbl 0473.53037

[10] R. Courant and D. Hilbert, Methoden der mathematischen Physik I. J. Springer, Berlin, 1931. English transl. Methods of mathematical physics I. Interscience Publishers, New York, 1953. MR 0065391 JFM 57.0245.01 Zbl 0053.02805

[11] E. N. Dancer, The effect of domain shape on the number of positive solutions of certain nonlinear equations. J. Differential Equations 74 (1988), 120-156. MR 0949628 Zbl 0662.34025

[12] E. N. Dancer, The effect of domain shape on the number of positive solutions of certain nonlinear equations II. J. Differential Equations 87 (1990), 316-339. MR 1072904 Zbl 0729.35050

[13] D. Daners, Dirichlet problems on varying domains. J. Differential Equations 188 (2003), 591-624. MR 1955096 Zbl 090.35069

[14] E. B. Davies, Eigenvalue stability bounds via weighted Sobolev spaces. Math Z. 214 (1993), 357-371. MR 1245198 Zbl 0799.58082 
[15] P. Exner and O. Post, Approximation of quantum graph vertex couplings by scaled Schrödinger operators on thin branched manifolds. J. Phys. A 42 (2009), Article Id. 415305. MR 2545638 Zbl 1179.81080

[16] F. W. Gehring, The $L^{p}$-integrability of the partial derivatives of a quasiconformal mapping. Acta Math. 130 (1973), 265-277. MR 0402038 Zbl 0258.30021

[17] M. Giaquinta, Multiple integrals in the calculus of variations and nonlinear elliptic systems. Annals of Mathematics Studies 105. Princeton Univ. Press, Princeton, NJ, 1983. MR 0717034 Zbl 0516.49003

[18] M. Giaquinta and G. Modica, Regularity results for some classes of higher order nonlinear elliptic systems. J. Reine Angew. Math. 311/312 (1979), 145-169. MR 0549962 Zbl 0409.35015

[19] D. Gilbarg and N. S. Trudinger, Elliptic partial differential equations of the second order. Reprint of the 1998 edition. Classics in Mathematics. Springer Verlag, Berlin, 2001. MR 1814364 Zbl 1042.35002

[20] D. Grieser and D. Jerison, Asymptotics of eigenfunctions on plane domains. Pacific J. Math 240 (2009), 109-133. MR 2485476 Zbl 1175.35006

[21] E. Hebey, Nonlinear analysis on manifolds: Sobolev spaces and inequalities. Courant Lecture Notes 5. Amer. Math. Soc., Providence, RI, 1999. MR 1688256 Zbl 0981.58006

[22] S. Jimbo, Perturbation formula of eigenvalues in a singularly perturbed domain. J. Math. Soc. Japan 45 (1993), 339-356. MR 1206658 Zbl 0785.35069

[23] S. Jimbo and S. Kosugi, Spectra of domains with partial degeneration. J. Math. Sci. Univ. Tokyo 16 (2009), 269-414. MR 2597393 Zbl 1231.35130

[24] S. Jimbo and Y. Morita, Remarks on the behavior of certain eigenvalues on a singularly perturbed domain with several thin channels. Comm. Partial Differential Equations 17 (1992), 523-552. MR 1163435 Zbl 0766.35029

[25] V. Kozlov, Domain dependence of eigenvalues of elliptic type operators. Preprint 2012. arXiv:1203.2093v1

[26] P. Kuchment, Graph models for waves in thin structures. Waves in Random Media 12 (2002), R1-R24. MR 1937279 Zbl 1063.35525

[27] O. A. Oleinik, A. S. Shamaev, and G. A. Yosifian, Mathematical problems in elasticity and homogenization. Studies in Mathematics and its Applications 26. North-Holland Publishing Co., Amsterdam, 1992. MR 1195131 Zbl 0768.73003

[28] M. M. H. Pang, Approximation of ground state eigenvalues and eigenfunctions of Dirichlet Laplacians. Bull. London Math. Soc. 29 (1997), 720-730. MR 1468060 Zbl 0969.35102

[29] J. Rauch and M. Taylor, Potential and scattering theory on wildly perturbed domains. J. Funct. Anal. 18 (1975), 27-59. MR 0377303 Zbl 0293.35056

[30] P. Shi and S. Wright, Higher integrability of the gradient in linear elasticity. Math Ann. 299 (1994), 435-448. MR 1282226 Zbl 0806.73009

[31] J. Taylor, Convergence of eigenvalues for elliptic systems on domains with thin tubes and the Green function for the mixed problem. Ph.D. Thesis. University of Kentucky, Lexington, KY, 2011. MR 2995915 
[32] F. Treves, Basic linear partial differential equations. Pure and Applied Mathematics 62. Academic Press, New York etc., 1975. Reprint Dover Publications, Mineola, NY, 2006. MR 0447753 Zbl 0305.35001 MR 2301309 (reprint) Zbl 1115.35006 (reprint)

Received March 19, 2012; revised July 26, 2012

Justin L. Taylor, Department of Mathematics and Statistics, Murray State University, Murray, Kentucky, U.S.A.

E-mail: jtaylor52@murraystate.edu 